The Geneva Papers on Risk and Insurance, 14 (No. 52, July 1989), 263-265

\title{
Response to Professor Robert C. Merton's Paper
}

\author{
by Brian Corby*
}

I approached this paper as an actuary and as a practitioner and I leave to others the task of commenting on Professor Merton's theoretical development; it is clearly well developed; I enjoyed reading it and I congratulate him on it. It does, however, lead to conclusions, with some at least of which I am not entirely comfortable.

The paper seems to have four main objectives:

1 To illustrate the fruitfulness of the continuous-time approach in yielding interesting theoretical results.

2 To demonstrate the practical relevance of continuous-time models for financial market practitioners and regulators.

3 To provide a theoretical rationale for the existence of financial intermediaries.

4 To consider the interaction of financial theory and financial practice.

As to theoretical fruitfulness, there can be no doubt that the continuous time approach has produced interesting results in relation to portfolio selection for the individual investor, to the evaluation of options and to work out an investment strategy to match an uncertain future liability.

What, however, I am not clear about is the extent to which similar results have been or could have been derived from a discrete time approach. That said, from my point of view the debate is not important - it is the results that count.

If I may digress for a moment into life insurance and actuarial theory. Actuaries have long struggled with problems of adequacy of reserves and equity between policyholders. Some two hundred years ago, at the beginning of life assurance as we now know it, the major problem - the major uncertainty if you like - was the rate of mortality. The solution adopted was to charge excessive premiums - of course they did not know they were excessive in advance - so that solvency was assured and then, when sufficient experience was accumulated to assess what the premiums should have been, to return the excess - or some of it - to policyholders by way of bonus additions. This was the origin of the traditional with profit policies we issue today. Equally those organisations which did not charge "excessive" premiums went out of business!

\footnotetext{
${ }^{*}$ Chief Executive, Prudential Corporation, London
} 
Over time mortality became more predictable - only to be changed very recently with the advent of AIDS - and with an increasing range of financial instruments becoming available, it was interest rate uncertainties which came to the fore. These were considered by Samuelson in the American Economic Review in 1945 and by Redington in the Journal of the Institute of Actuaries in 1952, who both came to similar conclusions based on the matching by average term of assets and liabilities. Redington used a continuous-time model with a solution varying continuously, and as with the binomial model he did not need to postulate an explicit model for future movements in interest rates.

Turning to the practical relevance of the theory, Professor Merton draws our attention correctly to several specific applications. But I suspect they are more in the field of guides to behaviour rather than hard and fast rules. In practice for an investor there is the underlying requirement to make instantaneous adjustments to the portfolio and I have some reservations as to its ability to deal fully with real world transaction costs. The approach of assuming zero transaction costs for the intermediary as opposed to the client, produces an elegant rationalisation of the role of the intermediary but leaves two questions which are answered satisfactorily only in the first instance.

First how fully can the intermediary protect its position? Only if it can do so fully will clients be willing to hold the derivative security it produces. Secondly, how is the equilibrium of agents buying derivative securities from such costless intermediaries related to the equilibrium, with which we are familiar, of a frictionless but risky world? The answer is "virtually identical", indeed strictly identical if the set of derived securities is rich enough, and Professor Merton has already shown that the number of required securities is reduced by certain trading strategies.

Is this too good to be true? Probably. Intermediaries can never act in a totally cost free way and therefore cannot afford continuous trading, quite apart from the question of practicality. Also there are important differences between a situation where investors trade derivative securities and one where they set out to achieve the same ends by an apparent equivalent trading strategy.

Returning to the more specific insurance world for a moment, continuous time models have been used in the United Kingdom to consider the level of the reserve required for maturity guarantees under variable type - unit linked - products. Despite the ability of the theory to set out a method whereby such products can be managed, it has been shown from the regulatory point of view that the level of reserves required is of such a high order as to make guarantees of this sort almost impossible to give, whatever the immunisation approach to the transaction of the business might conclude.

As to the rationale for the existence of financial intermediaries, it has to be said that some of the most fully developed and intellectually satisfying theories of financial markets are unable to provide an adequate interpretation of the role of the major participants such as insurance companies in real-world financial markets. Practitioners can have little time for theories that have no place for practitioners. In practice people don't take decisions based on probabilities but rather on an avoidance of risk - and they are prepared to pay for it.

Suffice to say here that we can take comfort from the fact that Professor Merton has managed to find a place for us intermediaries by postulating zero transactions cost - even though we and he know this not to be the case. 
Finally on the interaction between theory and practice. Mr Merton draws an analogy with the railways. If I really believed that it were possible so to improve the railway system that the high speed train would never either crash or rip up the track, I would feel much more comfortable than I do now. And I do not believe the regulators believe it either.

Nor do you have to believe that it was hyper-active trading policies of the sort indicated by continuous-time models which had a major part to play in bringing about the events of last October to come to that conslusion.

As Professor Merton says "the success of an intermediary depends not only on charging adequate prices to cover its production costs, but also on providing adequate assurances to its customers that promised payments will be made". He refers to investors with "unrealistic expectations of greater returns with less risk".

Without being specific on the particular policies, there are plenty of instances where, in order to achieve the return required by - or promised to - the investor, assets and liabilities are mismatched and for this reason, quite apart from the extent that guarantees and options are granted, the system will be unstable. If the managers of intermediaries offer investors the best of both worlds it is not surprising that the offer is accepted, but are the risks understood and should the regulators tolerate them?

A level of free reserves of solvency margin or whatever may be developed using a probabilistic approach - and should be - but at the end of the day what matters is that the investors' claims should be met. It is reasonable for the regulator to require intermediaries not only to be able to meet their claims but also to be able to demonstrate at all times that they can do so, given all the options and guarantees the investor may have been given. It is therefore reasonable for the regulator to ask the question of the intermediary "what if?" and to seek a satisfactory answer.

To end these remarks let me return to Professor Merton's train. Improving the infrastructure may give us an intellectually more interesting ride and a lot of exciting new routes to travel but it will not eliminate the risk. Indeed it could be that when the high speed train hits the buffers it will do so with even greater velocity. For the time being I'll go for some restriction on the types of trains we use, for the odd circuit breaker and a good hard look at the calibre of the engine drivers. 